\title{
Mode and wavelength-switchable pulsed fiber laser with few-mode fiber grating
}

\author{
Yu Cai, Zhiqiang Wang, Hongdan Wan, Zuxing Zhang, Lin Zhang
}

\begin{abstract}
We propose and demonstrate a mode and wavelength-switchable pulsed fiber laser using two-mode fiber Bragg grating (TM-FBG) as both transverse mode converter and wavelength selector. The mode-locking mechanism is based on saturable absorption of semiconductor saturable absorption mirror (SESAM). Due to the use of low polarization-dependent mode-locking method, polarization disturbation between mode-locking and transverse-mode selection has been effectively eliminated, thus enabling flexible transverse-mode selection with simultaneous mode-locking operation. Moreover, through the optimized offset launching technique, this laser can operate at the wavelengths of the fundamental mode (LP01) and second-order mode (LP11), and thus export cylindrical vector beams (CVBs) and fundamental mode beams at the TM-FBG output port, respectively. This all-fiber laser is a simple, low-cost and flexible source for mode-division multiplexing system and other applications.
\end{abstract}

Index Terms-Cylindrical vector beams, dual-wavelength, mode-locked lasers, two-mode fiber Bragg grating.

\section{INTRODUCTION}

I $\mathrm{n}$ recent years, the capacity of single-mode optical fiber transmission network is approaching the Shannon limit, due to the sharp increasing data demand driven by the Internet [1]. In order to further improve the capacity of the single-mode optical fiber transmission system and solve the bottleneck problem of increasingly tight bandwidth resource, the exploration of few- or multi-mode fibers supporting multiple independent transmission modes has become a promising solution, based on mode-division multiplexing (MDM) technology [2]. Therefore, it is critical to investigate fiber lasers with high-order mode emission, especially switchable dual-mode (fundamental and higher order modes) operation.

Manuscript received XXXX, 2019. This work was supported by Natural Science Foundation of Jiangsu Province (BK20161521 and BK20180742), Six Talent Peaks Project in Jiangsu Province (2015-XCL-023P), Postgraduate Research \& Practice Innovation Program of Jiangsu Province (KYCX17_0746), and the Talents Projects in Nanjing University of Posts and Telecommunications (NY214002, NY215002). (Corresponding authors: Zuxing Zhang).

Yu Cai, Zhiqiang Wang, Hongdan Wan, and Zuxing Zhang are with the Advanced Photonic Technology Lab, College of Electronic and Optical Engineering, Nanjing University of Posts and Telecommunications, Nanjing 210023, China.

Lin Zhang is with the Advanced Photonic Technology Lab, College of Electronic and Optical Engineering, Nanjing University of Posts and Telecommunications, Nanjing 210023, China and also with Aston Institute of Photonic Technologies, Aston University, Birmingham B4 7ET, UK (e-mail: 1.zhang@aston.ac.uk).
Besides being used for MDM transmission, few-mode fiber (FMF) has also important applications in mode manipulation through FMF-based devices, such as fiber taper [3,4], coupler [5-8], and grating [9-11]. Taking fiber Bragg grating as an example, since each mode of FMF is independent and has different propagation constant, the few-mode fiber Bragg grating (FM-FBG) can reflect different modes accommodated by the grating's phase matching condition and determined by the FMF spatial distribution. In the early days of their applications, FMF-FBGs were used for multiwavelength fiber lasers $[12,13]$, where each wavelength corresponds to different transverse modes. But the specific transverse mode was not figured out. Used as a mode convertor by controlling the wavelength of the desired mode, FM-FBG has been implemented to higher-order mode fiber laser. Under $\mathrm{CW}$ operation, the output mode of the laser can be selected to be LP01 mode, LP11 mode, or their mixing mode by adjusting the polarization state of light in the laser cavity [14-17]. This mode selection strategy has been also successfully applied to various types of mode-locked all-fiber lasers [18-20] and Q-switched fiber lasers [21]. For example, an all-fiber mode-locked laser producing radially and azimuthally polarized ultrafast pulses was demonstrated with carbon nanotube saturable absorber for longitude mode-locking and FM-FBG for transverse mode selecting [22]. Regarding Switchable dual-mode mode-locked fiber laser, Dong et al reported a mode-locked fiber laser based on nonlinear polarization rotation, generating stable picosecond pulses for both the LP01 and LP11 modes [23]. In this case, since both transverse mode section and mode-locking mechanism are polarization dependent, it may be challenging for simultaneous implementation of two functions.

In this letter, a mode and wavelength-switchable pulsed fiber laser using a TM-FBG has been proposed and demonstrated. Through optimizing the dislocation distance between the single- and two-mode fibers, separate laser operation on LP01 mode and LP11 mode has been obtained by appropriately adjusting the polarization controllers (PCs). The mode-locking mechanism is based on the saturated absorption of SESAM, which has low polarization dependent property and bring about reduced polarization disturbation between mode-locking and transverse mode selection. In addition, mode-locked operation at intercoupling mode has also been obtained with another dislocation distance. This provides us with a flexible way to control the laser output mode, and may find potential applications in mode-division multiplexing system and so on. 


\section{THE OFFSET SPLICING TECHNIQUE AND EXPERIMENT}

Offset splicing between the single-mode fiber (SMF) and single-mode fiber (TMF), as a kind of offset launch technique, is usually used to realize intermodal coupling and conversion between the LP01 and LP11 modes, as illustrated in Fig. 1. The LP01 mode is launched from the SMF, and the LP11 mode is expectedly excited at the offset splicing spot (OSS).

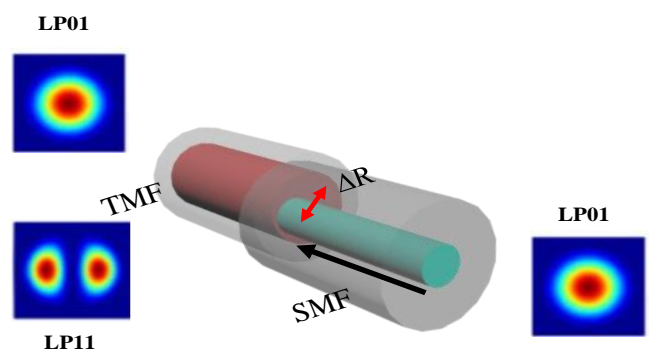

Fig. 1. Schematic of offset splicing between SMF and TMF. The $\mathrm{LP}_{01}$ mode is launched into the SMF input port, the $\mathrm{LP}_{11}$ mode is expected to be excited at the TMF.

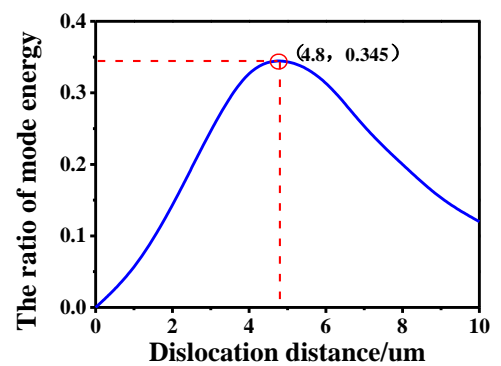

Fig. 2. Calculated ratio of LP01 and LP11 mode intensities varying with the dislocation distance.

In order to obtain the optimal dislocation distance for effective excitation of LP11 mode, the ratio $\alpha=E_{L P 11} / E_{L P 01}$ is introduced, $E_{L P 11}$ and $E_{L P 01}$ are the intensities of LP11 and LP01 modes in the TMF respectively. By using beam propagation method (BPM), the calculated ratio $\alpha$ varying with the dislocation distance is shown in Fig. 2. It is indicated that there exists an optimal dislocation distance, where the ratio $\alpha$ have a maximal value. Meanwhile, a TM-FBG was fabricated using phase mask scanning method with a $244 \mathrm{~nm}$ Argon-ion frequency-doubled laser. The length and maximum index modulation depth of the fabricated TM-FBG with grating period of $536 \mathrm{~nm}$ are estimated to be $10 \mathrm{~mm}$ and $2.5 \times 10^{-4}$, respectively. The reflection spectra of the TM-FBG under the different values of dislocation distance have been experimentally measured, as shown in Fig. 3. When there is no lateral misalignment, only rightmost reflection peak corresponding to the coupling between the forward- and backward-propagating LP01 appears. With the increasing dislocation distance, intermediate one corresponding to the intercoupling between the forward-propagating LP01 (LP11) mode and the backward-propagating LP11 (LP01) mode becomes stronger. Leftmost one corresponding to the coupling between the forward- and backward-propagating LP11 modes is ultimately excited under the condition of larger dislocation distance. This is to say that the three reflection peaks show the self-coupling of each mode (LP01 and LP11 modes) and the mutual coupling between LP01 and LP11 modes. The height of each reflection peak is related to the intensity ratio of each mode. When the intensity proportion of a mode increases, its self-coupling reflection peak increases. Therefore, for purpose of wavelength switching and mode conversion, it is necessary to select the dislocation distance corresponding to the maximal value of the ratio $\alpha$. Considering the possible deviation between theoretical calculation and measured results, the dislocation distance $5 \mu \mathrm{m}$ is chosen for switchable LP01 and LP11 dual-mode experiments, which can be accurately set in the fusion procedure.

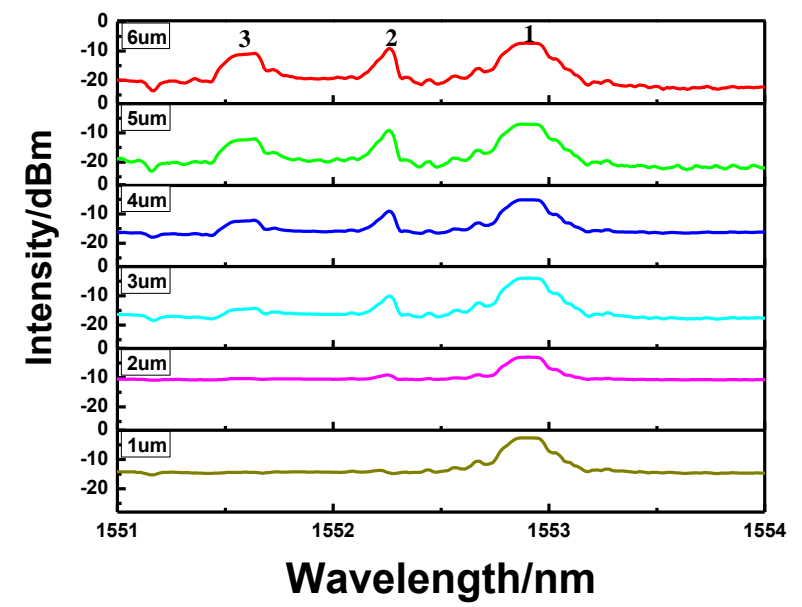

Fig. 3. Measured reflection spectra of the TM-FBG under the different values $(1 \sim 6 \mu \mathrm{m})$ of the dislocation distance.

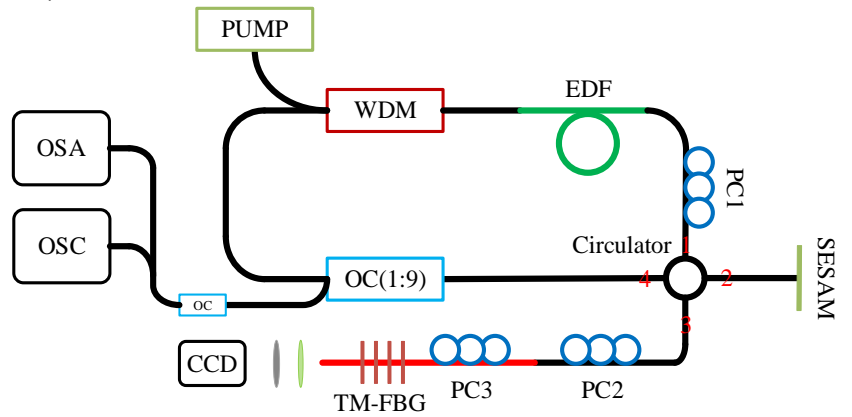

Fig. 4. Schematic setup of the proposed switchable dual-mode and dual-wavelength mode-locked fiber laser.

Fig. 4 shows the schematic of the proposed switchable dual-mode and dual-wavelength pulsed fiber laser. The total laser cavity length is around $13.5 \mathrm{~m}$, consisting of a $5 \mathrm{~m}$ erbium-doped fiber (EDF) with dispersion parameter D of -16 $\mathrm{ps} / \mathrm{nm} / \mathrm{km}$ at $1550 \mathrm{~nm}$, a $7.5 \mathrm{~m} \mathrm{SMF}$ with dispersion parameter D of $17 \mathrm{ps} / \mathrm{nm} / \mathrm{km}$ and a $1 \mathrm{~m} \mathrm{TMF}$ (from OFS) with inscribed TM-FBG. The dispersion D of the TMF for LP01 mode is 21.1 $\mathrm{ps} / \mathrm{nm} / \mathrm{km}$ at $1550 \mathrm{~nm}$. The calculated total dispersion of the laser cavity is about $-0.087 \mathrm{ps}^{\wedge} 2$. The SESAM and the TM-FBG are connected to the ring cavity via a four-port circulator. The light coming from the port 1 of four-port circulator is reflected through SESAM on the port 2, then the light enters into the port 3 for mode conversion and wavelength selection through OSS and TM-FBG respectively, and finally is fed back into the cavity via port 4 . The PCs are used to adjust the polarization states. The laser is pumped by a $980 \mathrm{~nm}$ laser diode (LD) through a wavelength division multiplexer (WDM). 
An optical spectrum analyzer (Yokogawa AQ-6370D), a radio-frequency analyzer (FSV30), and a digital storage oscilloscope (LeCroy SDA 6000A) with a photoelectric detector are used to monitor the laser output simultaneously from $10 \%$ output of the optical coupler (OC). The CVB is recorded by the CCD camera (CinCam IR) from the output of the TM-FBG.

\section{EXPERIMENT RESULTS AND DISCUSSION}

(a)

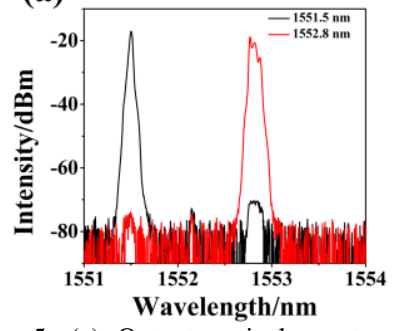

(b)

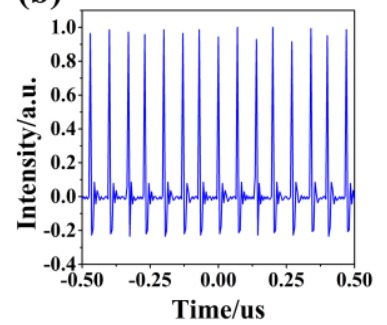

Fig. 5. (a) Output optical spectra with switchable wavelengths, and (b) mode-locked pulse train.

When the pump power is above the mode-locking threshold value of $150 \mathrm{mw}$, mode-locking can be easily established. The pump power was fixed at $200 \mathrm{~mW}$ throughout the whole experiment. It is found that the mode status is deeply affected by adjusting the PCs, while keeping the TM-FBG straight. Through adjusting the PCs, the mode-locking operation at the LP01 mode wavelength of $1552.8 \mathrm{~nm}$ and the LP11 mode wavelength of $1551.5 \mathrm{~nm}$ can be obtained respectively, as show in Fig. 5(a). The 3-dB linewidth of laser output spectrum for both modes is less than $0.02 \mathrm{~nm}$. Taking the mode-locking operation at the LP01 mode wavelength as an example, the mode-locked pulse train is shown in Fig. 5(b) and the repetition rate of the pulse corresponds to the fundamental rate related to the length cavity. Fig. 6(a) is the RF spectrum in wide span range (up to $1 \mathrm{GHz}$ ) and Fig. 6(b) is the RF spectrum in narrow range $(0.5 \mathrm{KHz})$ with a signal-to-noise ratio of about $49.5 \mathrm{~dB}$, indicating good stability of the mode-locking operation. The pulse duration is about $56.8 \mathrm{ps}$, as the autocorrelation trace shown in the inset of Fig. 6(b). The maximum output power from the TM-FBG port is $1.25 \mathrm{~mW}$ with pump power of 310.5 $\mathrm{mW}$. (a)

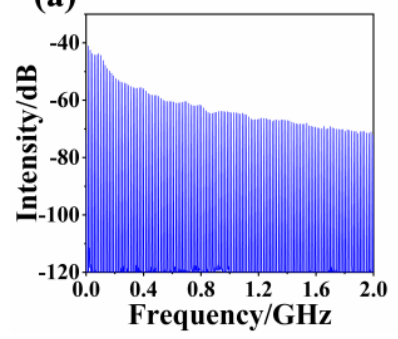

(b)

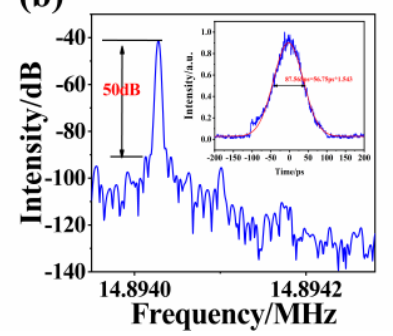

Fig. 6. RF spectra with range of (a) $2 \mathrm{GHz}$ and (b) $0.5 \mathrm{KHz}$ (the inset is the measured autocorrelation trace).

The TM-FBG reflects back the LP01 mode while exports the CVBs when the laser spectrum locates at the rightmost peak of the TM-FBG reflection spectrum, shown in Fig. 3(a) (Red line). Both radially (TM01) and azimuthally (TE01) polarized beams can be obtained via adjusting the PC2 and PC 3 , as showed in
Fig. 7(a) and (f), respectively. TM01 and TE01 can be distinguished by inserting linear polarizer between the collimator and the CCD camera. The arrows in Fig. 7(b-e) and $7(\mathrm{~g}-\mathrm{j})$ indicate the transmission directions of the polarizer. In the Fig. 7(b-e), two-lobe-shaped intensity patterns coincide with the direction of the liner polarizer, indicating that the generated beam was TM01. Conversely, when the two directions are perpendicular, the generated beam is TE01.

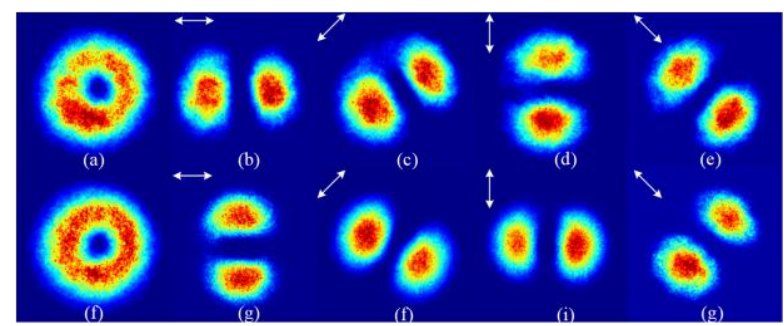

Fig. 7. Intensity distributions of TM01 (a) and TE01 (f) modes, and corresponding evolutions with rotation of the linear polarizer.

Using function fitting method, the mode purity can be estimated [19]. Figs. 8(a) and (b) show the typical function fitting curves of intensity distributions for the output TM01 and TE01 beams with calculated mode purities of $91.54 \%$ and $90.24 \%$, respectively. It can be seen that the intensity distributions in two lobes are almost symmetrical, while the intensity dip between the two lobes is near but not zero, indicating the existence of weak LP01 mode. It may be due to the incomplete reflection of LP01 mode (a)

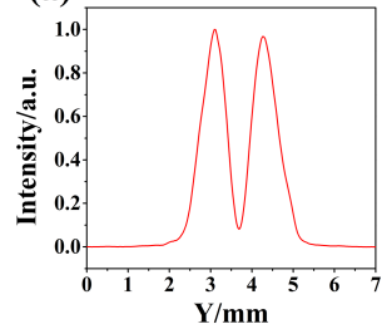

(b)

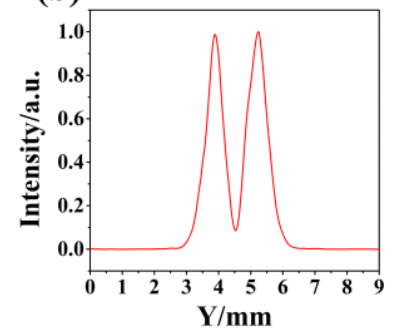

Fig. 8. Function fitting of output beam intensity distributions of (a) TM01 and (b) TE01 from the TM-FBG output port. (a)

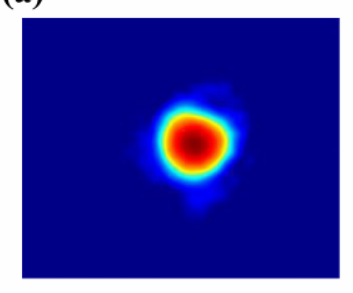

(b)

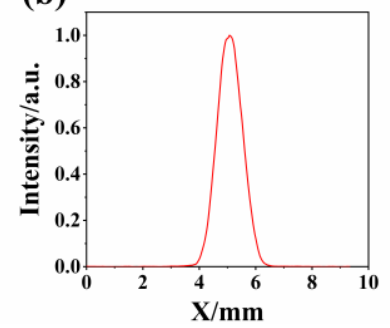

Fig. 9. (a) Intensity distribution and (b) function fitting of the LP01 mode from the TM-FBG output port.

The TM-FBG can act as a wavelength selector in this mode-locked fiber laser. As the PCs adjusted continually, the output laser spectrum can be tuned to the leftmost peak of the TM-FBG reflection spectrum, as black line shown in Fig. 3(a). The TM-FBG reflects back the LP11 mode, and the fundamental mode passes through the TM-FBG. Thus, the LP01 mode can be measured by the CCD at the end of the TM-FBG. The intensity distribution and function fitting of the LP01 mode are shown in the Fig. 9 (a) and (b). 
It has been numerically and experimentally validated that the reflection spectrum of the TM-FBG is seriously related the dislocation distance. It may pave a new way to manipulate laser output mode. Here, when the dislocation distance is changed to be $4.5 \mu \mathrm{m}$, the mode-locking oscillation operation with the LP01 mode at $1552.8 \mathrm{~nm}$ and the intercoupling mode at 1552.2 $\mathrm{nm}$ can be obtained respectively, as show in Fig. 10. When the oscillation wavelength is $1552.8 \mathrm{~nm}$ of LP01 mode, output beam from the TM-FBG output is LP11 mode, similar to the above case. Interestingly, when the oscillation wavelength is $1552.2 \mathrm{~nm}$ of the intercoupling mode, output beam from the TM-FBG output should be superposition of LP01 and LP11 modes [23].

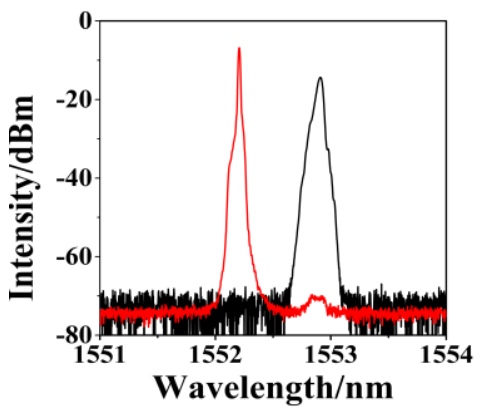

Fig. 10. Output spectra of LP01 mode and intercoupling mode, when the dislocation distance is $4.5 \mu \mathrm{m}$.

It should be noted that for our proposed mode and wavelength switchable mode-locked fiber laser with FM-FBG, the utilization of SESAM for mode-locking is very critical due to its polarization independence. Otherwise, polarization-dependent mode-locking mechanism, such as nonlinear polarization rotation [24], would bring about polarization disturbation with transverse-mode selection of the FM-FBG, since the reflection of the FM-FBG has great polarization dependence. This is to say that it is hard to simultaneously meet mode-locking and mode-selection conditions, if both them are polarization dependent. Additionally, different modes can be effectively excited through optimizing the dislocation distance between the SMF and TMF. Moreover, compared with direct mode conversion outside the laser cavity, the mode conversion in the laser cavity may lead to larger mode purity.

\section{Conclusion}

In conclusion, a switchable dual-wavelength and dual-mode mode-locked fiber laser has been demonstrated using TM-FBG as transverse mode converter and wavelength selector. It has been numerically and experimentally confirmed that the dislocation distance between the single and few-mode fibers has an important influence on the laser output mode. The mode-locking mechanism based on saturable absorption of SESAM with low polarization dependent property eliminating polarization disturbation between mode-locking and transverse-mode selection, enable controllable mode output with mode-locking operation. Switchable mode-locked operation on LP01 and LP11 modes has been obtained with optimal dislocation distance, in addition to operation at intercoupling mode. This all-fiber laser with simple and low-cost configuration is a flexible source suitable for mode-division multiplexing system and other applications.

\section{REFERENCES}

[1] R. J. Essiambre, G. Kramer, P. J. Winzer, G. J. Foschini, and B. Goebel, "Capacity Limits of Optical Fiber Networks," J. Lightwave Technol., vol. 28, no. 4, pp. 662-701, Feb. 2010.

[2] D. J. Richardson, J. M. Fini, and L. E. Nelson, "Space division multiplexing in optical fibres," Nat. Photonics, vol. 7, no. 5, pp. 354-362, Apr. 2013.

[3] V. R. Almeida, R. R. Panepucci, and L. Michal, "Nanotaper for compact mode conversion," Opt. Lett., vol. 28, no. 15, pp. 1302-1304, Aug. 2003.

[4] B. Sun, F. Fang, Z. Zhang, J. Xu, and L. Zhang, "High-sensitivity and low-temperature magnetic field sensor based on tapered two-mode fiber interference," Opt. Lett., vol. 43, no. 6, pp. 1311-1314, Mar. 2018.

[5] H. Wan, J. Wang, Z. Zhang, Y. Cai, B. Sun, and L. Zhang, "High efficiency mode-locked cylindrical vector beam fiber laser based on a mode selective coupler," Opt. Express, vol. 25, no. 10, pp. 11444-11451, May. 2017

[6] F. Wang, F. Shi, T. Wang, F. Pang, T. Wang, and X. Zeng, "Method of Generating Femtosecond Cylindrical Vector Beams Using Broadband Mode Converter," IEEE Photonic Tech. L., vol. 29, no. 9, pp. 747-750, May. 2017.

[7] Y. Shen et al., "Generation of the Tunable Second-Order Optical Vortex Beams in Narrow Linewidth Fiber Laser," IEEE Photonic Tech. L., vol. 29, no. 19, pp. 1659-1662, Aug. 2017.

[8] D. Mao et al., "All-fiber radially/azimuthally polarized lasers based on mode coupling of tapered fibers," Opt. Lett., vol. 43, no. 7, pp. 1590-1593, Apr. 2018.

[9] J. Dong, and K. Chiang, "Temperature-Insensitive Mode Converters With CO2-Laser Written Long-Period Fiber Gratings," IEEE Photonic Tech. L., vol. 27, no. 9, pp. 1006-1009, May. 2015.

[10] Y. Zhao, Y. Liu, C. Zhang, L. Zhang, G. Zheng, C. Mou, J. Wen, and T. Wang, "All-fiber mode converter based on long-period fiber gratings written in few-mode fiber," Opt. Lett., vol. 42, no. 22, pp. 4708-4711, Nov. 2017.

[11]Y. Han et al., "Orbital angular momentum transition of light using a cylindrical vector beam," Opt. Lett., vol. 43, no. 9, pp. 2146-2149, Apr. 2018.

[12]X. Feng et al., "Switchable dual-wavelength ytterbium-doped fiber laser based on a few-mode fiber grating," IEEE Photonic Tech. L., vol. 16, no. 3, pp. 762-764, Mar. 2004.

[13] Y. G. Han, S. B. Lee, D. S. Moon and Y. Chung, "Investigation of a multiwavelength raman fiber laser based on few-mode fiber Bragg gratings," Opt. Lett., vol. 30, no. 17, pp. 2200-2202, Sep. 2005.

[14]B. Sun et al., "Low-threshold single-wavelength all-fiber laser generating cylindrical vector beams using a few-mode fiber Bragg grating," Opt. Lett., vol. 37, no. 4, pp. 464-466, Feb. 2012.

[15]B. Sun et al., "Transverse mode switchable fiber laser through wavelength tuning," Opt. Lett., vol. 38, no.5, pp. 667-669, Mar. 2013.

[16] W. Jin et al., "Switchable dual-mode all-fiber laser with few-mode fiber Bragg grating," J. of Opt., vol. 19, no. 9, pp. 095702, Aug. 2017.

[17]Y. Shen et al., "Switchable narrow linewidth fiber laser with LP11 transverse mode output," Opt. Laser. Technol., vol.98, pp. 1-6, Jan. 2018.

[18]B. Sun et al., "Mode-locked all-fiber laser producing radially polarized rectangular pulses," Opt. Lett., vol. 40, no. 8, pp. 1691-1694, Apr. 2015.

[19] Y. Zhou et al., "Self-starting passively mode-locked all fiber laser based on carbon nanotubes with radially polarized emission," Photon. Res., vol. 4, no. 6, pp. 327-330, Dec. 2016.

[20] Y. Zhou et al., "Actively mode-locked all fiber laser with cylindrical vector beam output," Opt. Lett., vol. 41, no. 3, pp. 548-550, Feb. 2016.

[21] K. Yan, J. Lin, Y. Zhou, C. Gu, L. Xu, A. Wang, P. Yao, and Q. Zhan, "Bi2Te3 based passively Q-switched fiber laser with cylindrical vector beam emission," Appl. Optics, vol. 55, no. 11, pp. 3026-3029, Apr. 2016.

[22]D. Mao et al., "Ultrafast all-fiber based cylindrical-vector beam laser," Appl. Phys. Lett., vol. 110, no. 2, pp. 021107, Jan. 2017.

[23] Y. Cai, Z. Wang, et al., "Switchable dual-mode pulsed fiber laser with few-mode fiber grating," in CLEO Pacific Rim Conference 2018, OSA Technical Digest (Optical Society of America, 2018), paper Th2A.2.

[24]J. Dong, and K. S. Chiang. "Mode-Locked Fiber Laser With Transverse-Mode Selection Based on a Two-Mode FBG," IEEE Photonic Tech. Lett., vol. 26, no. 17, pp. 1766-1769, Jul. 2014. 